\title{
A BUFF-YELLOW SPORE MUTANT COMBINATION IN SORDARIA BREVICOLLIS
}

\author{
M.H.V. COORAY \\ Botany School, Cambridge, England.
}

(Date of receipt : 27 April 1993)

(Date of acceptance : 20 August 1993)

\begin{abstract}
A buff-yellow spore mutant combination in :Sordaria brevicollis, where all four individual spore genotypes and all three non-aberrant tetrad types namely parental ditypes, non-parental ditypes and tetratypes - can be directly detected and scored under the microscope, is described. Aberrant segregation octads too are directly and easily detected in this system.
\end{abstract}

Key words: Aberrant segregation, mutant, Sordaria brevicollis, spores.

S. brevicollis is a heterothallic fungus isolated and described by Olive \& Fantini. ${ }^{1}$

In this laboratory a number of spore mutants of this fungus has been isolated, all UV induced. One millilitre of a microconidial suspension of density $5 \times 10^{6}$ per ml in distilled water was pipetted into a jena glass tube and treated with UV for one minute. The UV was from a mercury vapour lamp T/M5/369 supplied by the Thermal Syndicate Ltd. It was a low presure lamp running at about $110 \mathrm{~m}$.a. with an arc voltage of 300 to 350. (kill of E.coli strain B at $18 \mathrm{~cm}$ suggests an intensity of 10,000 to 12,000 ergs $/ \mathrm{cm}^{2} / \mathrm{sec}$ ). The UV treatment was carried out at $20^{0} \mathrm{C}$.

A number of mutants are buff in colour, e.g. $S_{6}, S_{42}, S_{44}, S_{140}, S_{155}$ and $S_{156}$ : they show extremely low recombination frequencies amongst them and all of these buff map at the bei-1 locus . Another five markers, namely $S_{27}, S_{62}, S_{150}, S_{187}$ and $S_{214}$ are all yellow in colour, show extremely low recombination frequencies amongst them and they all map at the ylo-9 locus. ${ }^{2}$ Hence these eleven mutants are all in linkage group II. The buff mutants, the yellow mutants, the double mutant (white) and the wild type (dark brown) can all be easily detected under the light microscope at a magnification of 400 .

An interesting feature of these mutants is that in any buff $x$ yellow cross, e.g. $\mathrm{S}_{6} \times \mathrm{S}_{27}$, in a non-aberrant recombination ascus, two black (wild type) spores are invariably accompanied by two white spores; four black spores are invariably accompaied by four white spores. Preliminary counts were compatible with the assumption that a two buff, two yellow, two black and two white ascus (plate 2) was a tetratype and the assumption that a four black and four white ascus (plate 3) was a non-parental ditype. Hence the white spore had to be the double mutant. In later work these white spores were isolated, germinated, back crossed to the wild type and confirmed to be the double mutants.

* Present address: Department of Botany, University of Peradeniya, Peradeniya. 


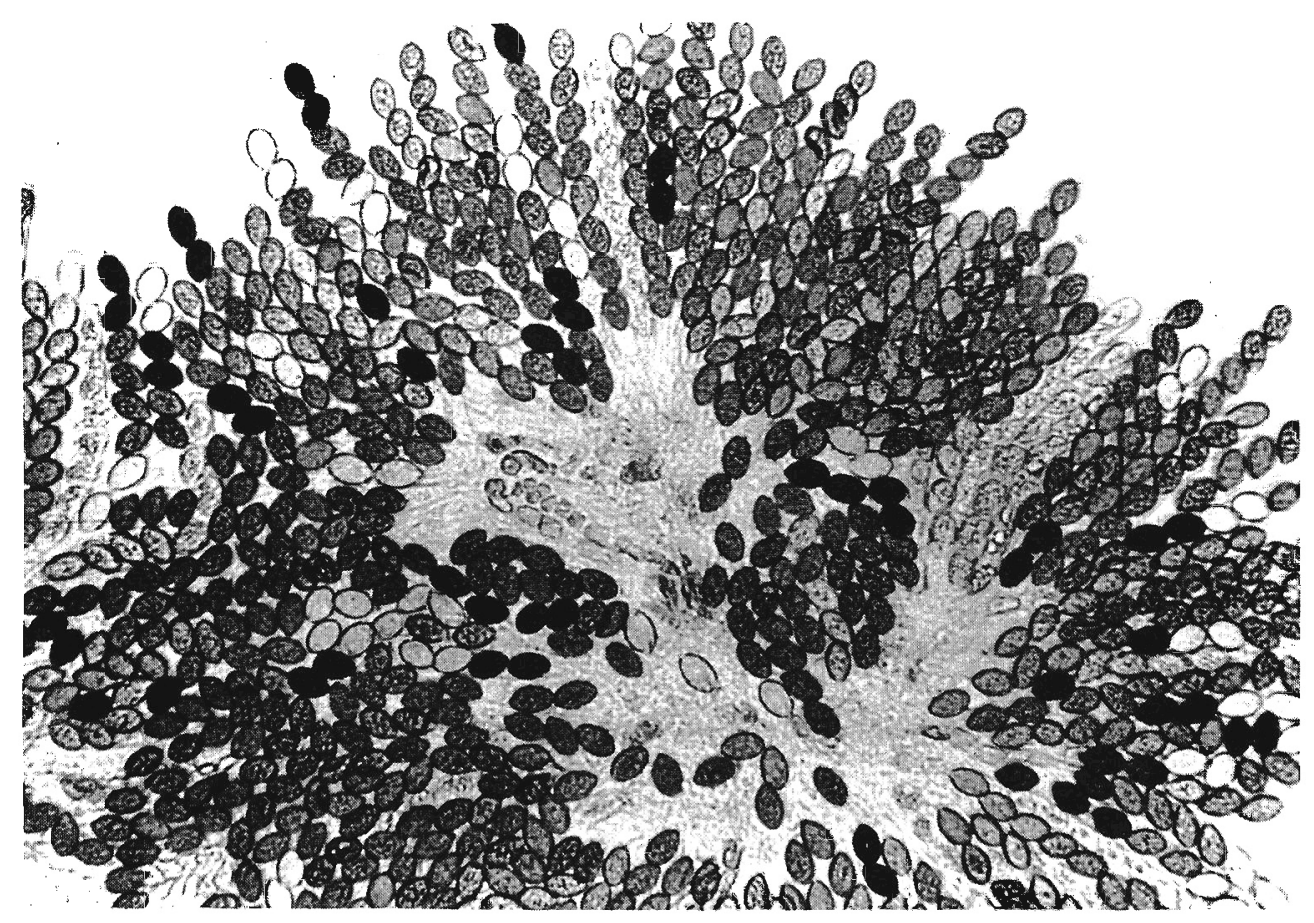

Plate 1: A cluster of asci from a buff $x$ yellow cross. Magnification ca x 260 .

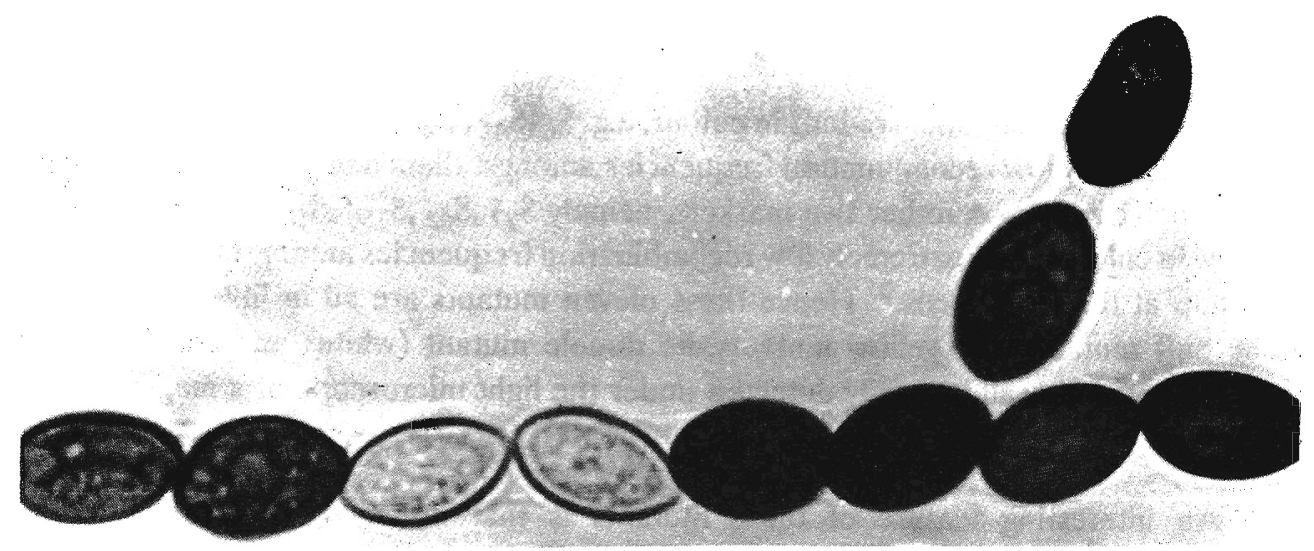

Plate 2: A tetratype ascus from a buff x yellow cross. Magnification ca $x 1280$

Hence this buff-yellow combination affords a unique system where all four individual spore genotypes and all three non-aberrant tetrad types can be directly detected and scored under the microscope. This system affords a unique opportunity of demonstrating the phenomena of mutation, segregation, linkage and recombination and the idea that only two of the four chromatids are involved in a particular recombination event, all under the microscope (plate 1), e.g. in undergraduate practical classes. 


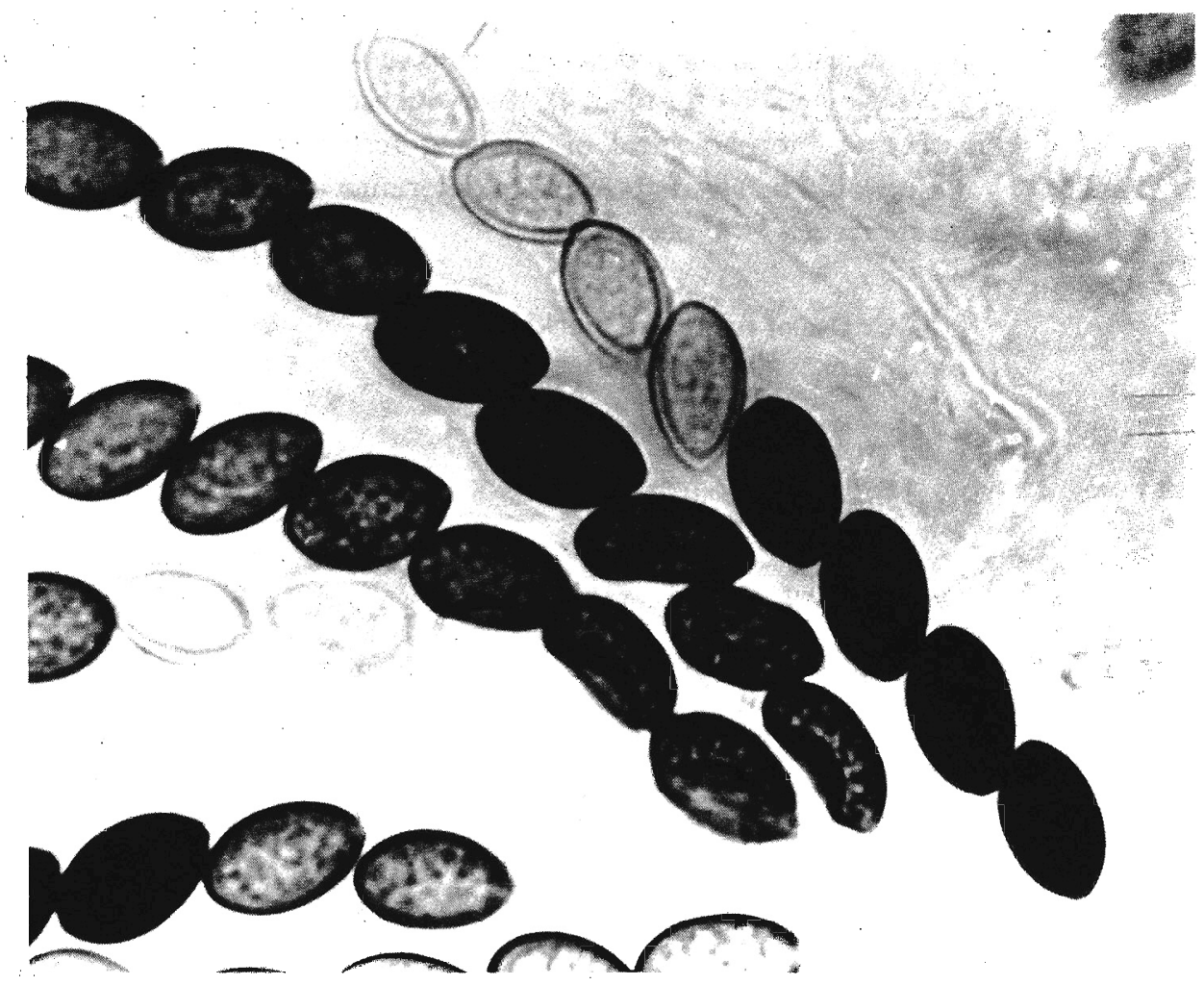

Plate 3: A non-parental ditype ascus from a buff $x$ yellow cross. Magnification ca x 1280.

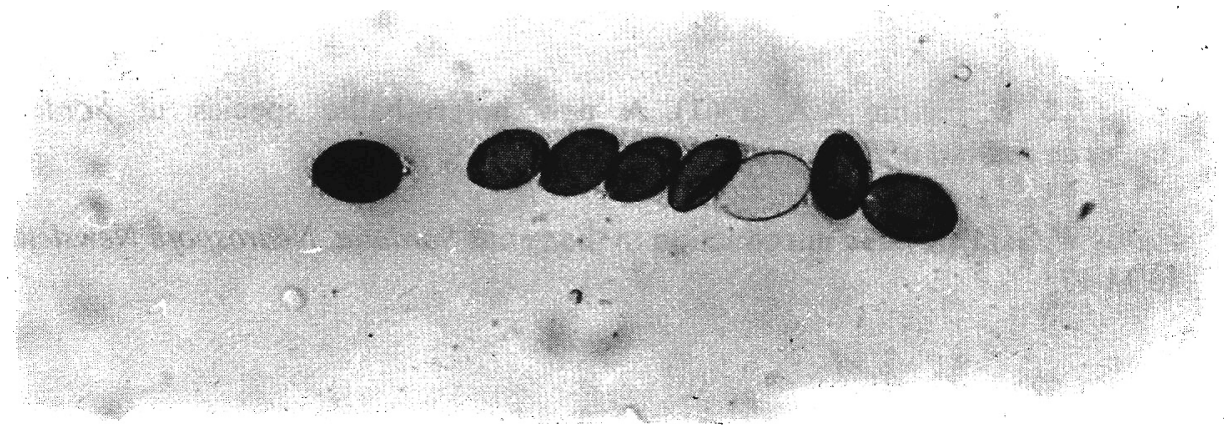

Plate 4: An aberrant $4^{+}: 4^{-}$ascus from a bufr $x$ yellow cross. Magnification ca $x 640$.

In addition it is found that each of the aberrant segregation octads can be easily detected. It is not always easy to determine at which of the two loci (buff or yellow) the aberrant segregation occurred as it is sometimes difficult to judge whether the odd spore is yellow or buff.

An aberrant $4^{+}: 4^{-}$octad is easily detected in this system as such an octad will have three buff, three yellow, one black and one white spore (plate 4). Here again it is not 
always possible to say whether the aberrant segregation occurred at the buff or yellow locus. Sometime it is possible to track down the locus at which the aberrant $4^{+}: 4^{-}$ occurred (see Fig. 1 as explanation for plate 4). But with ascus dissection and the use of an outside marker (a morphological $\mathrm{m}^{-}$showing restricted growth) available proximal to the buff it would be always possible to determine at which of the two loci (buff or yellow) the aberrant $4^{+} 4^{-}$occurred.

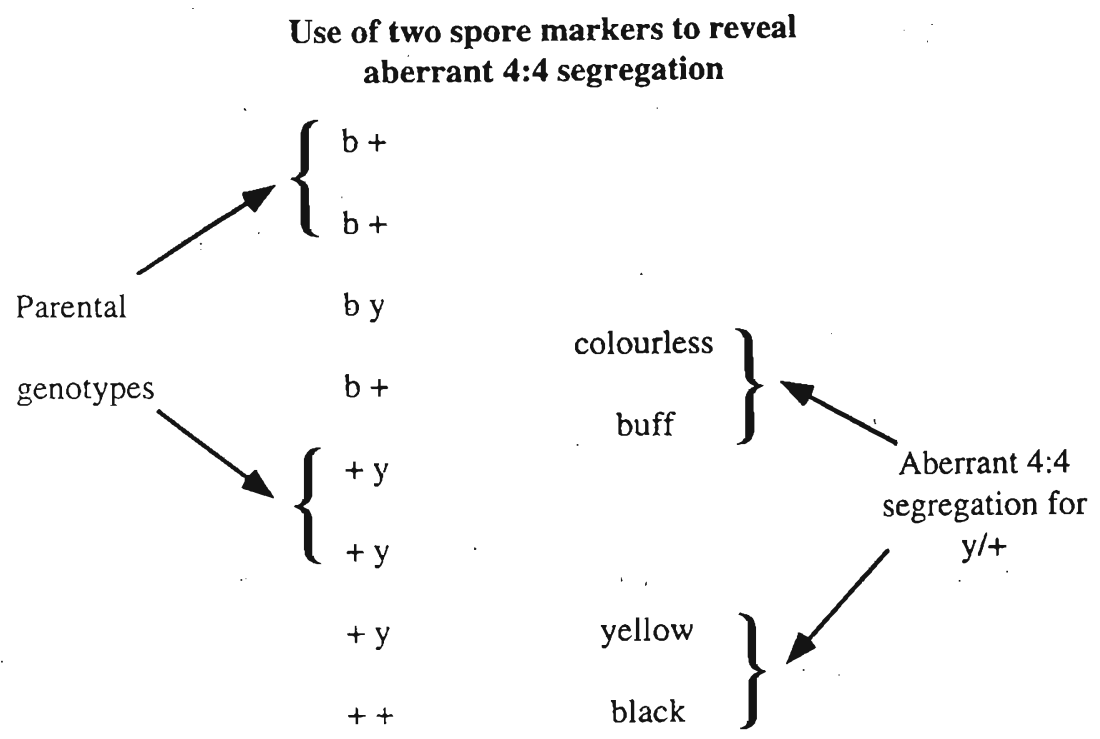

Figure 1: Use of two spore markers to reveal aberrant $4^{+}: 4^{-}$segregation. An explanation for plate 4.

\section{References}

1. Olive L.S. \& Fantini A.A. (1961). A new heterothallic species of Sordaria. American Joumal of Botany $48: 124-128$.

2. Fields W.G. (1970). An introduction to the genus Sordaria. Neurospora Newsletter 16:14-17. 